\title{
Prevalence of Fructose Malabsorption in Patients With Irritable Bowel Syndrome After Excluding Small Intestinal Bacterial Overgrowth
}

\author{
Kee Wook Jung, ${ }^{1}$ Myeognsook Seo, ${ }^{1}$ Young Hwan Cho, ${ }^{1}$ Young-Ok Park, ${ }^{2}$ So-Yoon Yoon, ${ }^{2}$ Jungbok Lee, ${ }^{3}$ Dong-Hoon Yang, ${ }^{1}$ \\ In Ja Yoon, ${ }^{1}$ So Young Seo, ${ }^{1}$ Hyo Jeong Lee, ${ }^{1}$ Sang Hyoung Park, ${ }^{1}$ Kyung Jo Kim, ${ }^{1}$ Byong Duk Ye, ${ }^{1}$ Jeong-Sik Byeon, ${ }^{1}$ \\ Hwoon-Yong Jung, ${ }^{1}$ Suk-Kyun Yang, ${ }^{1}$ Jin-Ho Kim, ${ }^{1}$ and Seung-Jae Myung ${ }^{1 *}$ \\ Departments of ${ }^{1}$ Gastroenterology, ${ }^{2}$ Clinical Nutrition, and ${ }^{3}$ Clinical Epidemiology and Biostatistics, Asan Medical Center, University of Ulsan \\ College of Medicine, Seoul, Korea
}

\section{Background/Aims}

Fructose malabsorption (FM) mimics symptoms of irritable bowel syndrome (IBS), and its prevalence has increased. Diagnosing FM in IBS is challenging because of its overlap with small intestinal bacterial overgrowth (SIBO). We assessed the prevalence of FM by comparing patients with IBS with asymptomatic control individuals after excluding SIBO using the glucose hydrogen breath test (HBT).

\section{Methods}

Patients diagnosed with IBS and asymptomatic control individuals were enrolled prospectively. Dietary habits were assessed with the Food Frequency Questionnaire. After excluding SIBO, participants underwent HBTs with both $15 \mathrm{~g}$ and $25 \mathrm{~g}$ of fructose.

\section{Results}

Thirty-five patients with IBS and 35 age- and sex-matched asymptomatic control individuals were enrolled. The 15-g fructose HBT yielded positive results in 7 of the $35(20.0 \%)$ patients with IBS and in 2 of $35(5.7 \%)$ controls $(P=0.070)$. The 25 -g fructose HBT was positive in 16 of the $35(45.7 \%)$ patients with IBS and in 8 of the $35(22.9 \%)$ controls $(P=0.040)$. Analysis of the Food Frequency Questionnaire responses showed no significant differences between the 2 groups in dietary intake, although patients with IBS showed a significantly higher mean fiber intake than controls $(21.24 \pm 11.35 \mathrm{~g}$ vs $15.87 \pm 7.07 \mathrm{~g}$, respectively, $P=0.040)$.

\section{Conclusions}

The 25-g fructose HBT identified FM in a significantly higher percentage of SIBO-negative patients with IBS than in asymptomatic control individuals, suggesting that FM may correlate with IBS. Education regarding dietary control of foods containing fructose may be useful for the management of patients with IBS.

(J Neurogastroenterol Motil 2018;24:307-316)

Key Words

Breath tests; Dietary therapy; Fructose; Irritable bowel syndrome

Received: March 20, 2017 Revised: September 7, 2017 Accepted: October 13, 2017

(a) This is an Open Access article distributed under the terms of the Creative Commons Attribution Non-Commercial License (http://creativecommons. org/licenses/by-nc/4.0) which permits unrestricted non-commercial use, distribution, and reproduction in any medium, provided the original work is properly cited.

${ }^{*}$ Correspondence: Seung-Jae Myung, MD, PhD

Department of Gastroenterology, Asan Medical Center, University of Ulsan College of Medicine, 88 Olympic-ro 43-gil, Songpa-gu, Seoul 05505, Korea

Tel: +82-2-3010-3917, Fax: +82-2-3010-0824, E-mail: sjmyung@amc.seoul.kr

Kee Wook Jung and Myeongsook Seo contributed equally to this article. 


\section{Introduction}

Fructose is made of a hexose sugar that is excessively used in Western diets. ${ }^{1}$ A United States study shows fructose has replaced traditional cane sugar over the last several years, and that fructose is often used under the name of an artificial sweetener or in highfructose corn syrup (HFCS), and is present in many prepared and instant foods worldwide. ${ }^{2}$ Changes in dietary habits, from eating traditional healthy foods to consuming westernized fast foods, have increased the consumption of fructose and HFCS in Asian countries, including Korea.,

Ingested fructose is usually absorbed in the small bowel through glucose transporter-5 transporter-mediated facilitative diffusion. Because this is an energy-independent process, it limits the absorptive capacity of the small intestine carrier. ${ }^{5}$ Glucose increases intestinal fructose absorption through passive diffusion as well as the solvent dragging effect. ${ }^{6,7}$ However, because fructose is a monosaccharide, excessive dietary intake can easily exceed the absorptive capacity of the small bowel, leading to incomplete absorption of fructose and, finally, causing fructose malabsorption (FM). ${ }^{1}$ The unabsorbed fructose may play a role in osmotic load and is therefore rapidly propelled into the colon, where its contact with anaerobic microbiomes causes fermentation and the production of gaseous feeling, abdominal bloating, and even diarrhea. ${ }^{8,9}$ Because these symptoms are very similar to functional gastrointestinal disorders, including irritable bowel syndrome (IBS), the relationship between FM and IBS has been studied. ${ }^{7}$ However, the results have been equivocal, with the prevalence of FM in patients with IBS varying according to study designs.

Hydrogen breath testing (HBT), which detects end-expiratory alveolar hydrogen or methane gases, has been used to diagnose FM. ${ }^{1,8}$ A high percentage of patients with IBS have small intestinal bacterial overgrowth (SIBO), which is also diagnosed by $\mathrm{HBT}^{10}$ However, HBT results from individuals with SIBO depend on hydrogen or methane gas concentrations. ${ }^{11}$ Since patients with SIBO have abnormal bacterial overgrowths in their small intestines, fructose ingested during HBT for the diagnosis of FM can be fermented by these small intestinal bacteria, leading to the misdiagnosis of FM. ${ }^{8}$ Therefore, it is necessary to exclude SIBO to assess the prevalence of FM in patients with IBS. Moreover, no studies have compared the prevalence of FM and dietary habits between patients with IBS and asymptomatic control individuals after the exclusion of SIBO. In addition, the ideal amount of fructose that should be used in the HBT for diagnosis of FM is unclear, ${ }^{8,11}$ and the optimal fructose cut-off dose for Asian populations has not been fully evaluated.

Therefore, we evaluated the prevalence of FM in patients with IBS and asymptomatic control individuals after excluding SIBO using glucose HBT. In addition, we analyzed differences in dietary habits between the 2 groups. We also assessed the optimal fructose dose for HBT in the Asian population.

\section{Materials and Methods}

\section{Study Participants}

From December 2011 until November 2012, asymptomatic control individuals with no previous history of gastrointestinal disorders, surgeries, or antibiotic use (within 3 months), and who were not taking any medications (except oral contraceptive pills or multivitamins) were recruited through hospital and internet advertisements. Asymptomatic control individuals were administered Rome III questionnaires to exclude any possible symptomatic gastrointestinal disorders. ${ }^{12}$ Only subjects who were asymptomatic and had a normal physical examination were eligible to participate. To assess the effects of age and gender on difference between IBS and asymptomatic control individuals, attempts were made to match the age and gender of the participants by decade in 2 groups.

At the same time, among SIBO negative patients, IBS patients who visited a tertiary care center were identified using the Rome III questionnaire. They were prospectively enrolled in this study with informed consent. To reduce possible bias, IBS patients with a previous history of antibiotic use (within 3 months) were excluded. The exclusion criteria for both asymptomatic normal individuals and IBS patients also included abnormal findings on colonoscopy or barium enema (such as cancer, ulcer, or colitis); history of abdominal surgery (except for appendectomy) or anorectal surgery; significant cardiovascular, respiratory, neurological, endocrine, hepatic, or renal disease; the use of medications likely to affect gastrointestinal tract motility; and pregnancy.

\section{Dietary Habits}

The usual dietary intake of all participants was assessed with a validated self-administered Food Frequency Questionnaire (FFQ) and the responses were analyzed using a computer-aided nutritional analysis program. ${ }^{13,14}$ The FFQ included questions about the types of foods frequently consumed by Korean people that were included as specific categories, as well as about the specific amounts consumed and the frequencies at which each food was eaten. The FFQ 
is composed of 103 food items and incorporated 410 types of food commonly consumed by the enrolled subjects. All enrolled subjects were interviewed about average intake frequencies and portion sizes of specific foods they had eaten. Nine categories of frequency (never or rarely, once a month, twice or 3 times a month, once or twice a week, 3 or 4 times a week, 5 or 6 times a week, once a day, twice a day, and 3 times a day) and 3 portion sizes (small, medium, and large) were categorized in the FFQ. Food photographs with the usual intake portions were used to help participants estimate and record the amounts of food consumed.

A dietician (Y-O.P.) entered all responses into the computeraided nutritional analysis program. The program automatically analyzed the intakes of each participant, including total calories, proteins (animal and vegetable), lipids (animal and vegetable), fiber, carbohydrates, vitamins, cholesterol, amino acids, and other trace nutrients. Fructose-rich foods derived from the validated FFQ were analyzed separately and additionally as "fructose" and "fructose/carbohydrate."

\section{Study Protocol}

The study involved 3 visits to the motility laboratory at weekly intervals. Subjects were administered bowel symptom questionnaires, on which they recorded their baseline symptoms. On the day before each visit, subjects were asked to consume a lactose-free and fructose-restricted diet to avoid high baseline values of breath hydrogen or methane concentrations. To minimize false-positive results, no food or drink was allowed for at least 8 hours before each test. All enrolled participants were asked to brush their teeth before the test and to abstain from smoking, drinking, sleeping, or exercising during the test.

On the first visit, all participants were administered $75 \mathrm{~g}$ glucose HBTs. Two breath samples were taken at baseline, and samples were taken every 20 minutes for 3 hours. All breath samples were end-expiratory breath samples to ensure alveolar gas sampling. All the samples were analyzed soon after collection, with both hydrogen and methane concentrations measured using a gas chromatography analyzer (Microlyzer Model SC; QuinTron Instrument Company, Milwaukee, WI, USA). Participants with baseline hydrogen or methane concentrations below 10 parts per million (ppm) were considered positive for SIBO if their hydrogen or methane peak concentrations were above 12 ppm. ${ }^{11,15}$ Otherwise, subjects whose baseline hydrogen or methane concentrations above 10 ppm were considered positive for SIBO if their hydrogen or methane peak concentrations increased by more than 12 ppm. ${ }^{11,15}$ Participants with positive SIBO HBT results were excluded from this study.
After excluding subjects with SIBO, the enrolled participants underwent HBTs with both $15 \mathrm{~g}$ and $25 \mathrm{~g}$ of fructose (dissolved in $250 \mathrm{~mL}$ of water), with an interval of at least 1 week between tests. The solutions were kept at room temperature, and the subjects were asked to drink them within 10 minutes. ${ }^{8,16}$ The subject, and the individual who collected and analyzed the breath samples were blinded to the type of solution consumed. End-expiratory breath samples were collected into modified (Haldane-Priestley) bags (QuinTron) at 30-minute intervals for 3 hours. A $20 \mathrm{~mL}$ sample of air was withdrawn from the bag and injected into the gas chromatography analyzer to detect hydrogen and methane. ${ }^{8,16}$ If subjects experienced any symptoms - including belching, cramping, abdominal pain, diarrhea, fullness, bloating, vomiting, or flatulence - after ingestion of the fructose, its severity was documented on a visual analogue scale. The study protocol was approved by the institutional review board of the Asan Medical Center, Seoul, Korea, (No. 2011-0524) and all subjects provided written informed consent.

\section{Measurements and Analysis}

Hydrogen and methane concentrations in breath samples were analyzed, with FM defined as a sustained increase of $>20 \mathrm{ppm}$ of hydrogen or $>10$ ppm of methane or $>15$ ppm of hydrogen + methane over baseline, or an increase of $\geq 5 \mathrm{ppm}$ of hydrogen and/ or methane sustained in at least 3 consecutive breath samples taken within 3 hours. ${ }^{8,16}$ By plotting the breath-hydrogen and breathmethane values over time, we could assess the areas under the breath-hydrogen and breath-methane curves for each subject and for each fructose concentration. ${ }^{8}$ Assessments of the areas under the curves were not used to define positive test results, However, they provided semiquantitative assessments of the overall volumes of gas produced and FM indices. ${ }^{8}$

Onset times were determined as the interval between fructose ingestion and the onset of sustained increases in breath hydrogen and/or methane concentrations. ${ }^{8}$ The peak time was determined as the time interval between fructose ingestion and the occurrence of the peak breath hydrogen or methane concentrations. ${ }^{8}$

\section{Sample Size Calculation}

Although none of these studies were performed in Asian countries, previous studies have reported that $35-73 \%$ of patients with IBS or functional gastrointestinal disorders have FM. ${ }^{1,9,17}$ The exact prevalence of FM in asymptomatic control individuals has not been fully evaluated, even in westernized countries. Assuming that $45 \%$ of Korean patients with IBS have FM and that there is a $30 \%$ difference in FM prevalence between patients who have IBS and 
asymptomatic control individuals, the minimum number of subjects required in each group was estimated to be 34 (alpha $=0.05$; statistical power $=80 \%$ ). Considering a $20 \%$ dropout rate, 43 subjects were recruited into each group.

\section{Statistical Methods}

All data analyses were performed using SPSS Statistics 18.0 software package for Windows (IBM, New York, USA) with significance set at $P<0.05$. Differences between patient groups were determined using Student's $t$ test for continuous variables. Chisquare and Fisher's exact tests were used to determine differences in proportions for categorical variables.

\section{Results}

From December 2011 until November 2012, 220 patients underwent HBT in our center. Among them, 22 underwent HBT after ingesting $15 \mathrm{~g}$ of lactulose because of underlying diabetes mellitus. One hundred ninety-eight patients underwent HBT after ingesting $75 \mathrm{~g}$ of glucose. Ninety-six patients out of 198 (48.5\%) showed positive SIBO results based on 75-g glucose HBT. Among 102 patients with negative SIBO results, 42 patients with IBS were prospectively enrolled in this study (Fig. 1). After a dropout of 7 patients, 35 patients with IBS (male:female $[\mathrm{M}: \mathrm{F}]$ ratio $=16: 19$; mean age $=43 \pm 14$ years) were enrolled in the study. In addition, 35 asymptomatic control individuals $(\mathrm{M}: \mathrm{F}$ ratio $=18: 17$; mean age $=38 \pm 11$ years) were enrolled in the study after the exclusion of 2 SIBO-positive individuals and 9 individuals who dropped out. There were no significant between-group differences in relation to sex and age (Table 1). Among the individuals with IBS, 10 (29\%) had IBS with constipation (IBS-C), 18 (51\%) had IBS with diarrhea (IBS-D), and 7 (20\%) had mixed IBS (IBS-M).

\section{Hydrogen Breath Testing With 15-g Fructose}

The $15-\mathrm{g}$ fructose HBT yielded positive results in 7 of the 35 (20.0\%) SIBO-negative patients with IBS, and in 2 of the $35(5.7 \%)$ asymptomatic control individuals $(P=0.070)$ (Table 1, Fig. 2 and $3)$. There were no significant differences between the groups in relation to the timing of the onset of sustained increases in breath hydrogen and methane concentrations or the peak breath hydrogen and methane concentrations. During the test, the numbers of individuals reporting some of the symptoms associated with FM were significantly higher in the IBS group than in the control group, and these symptoms included abdominal pain, bloating, fullness, belch-

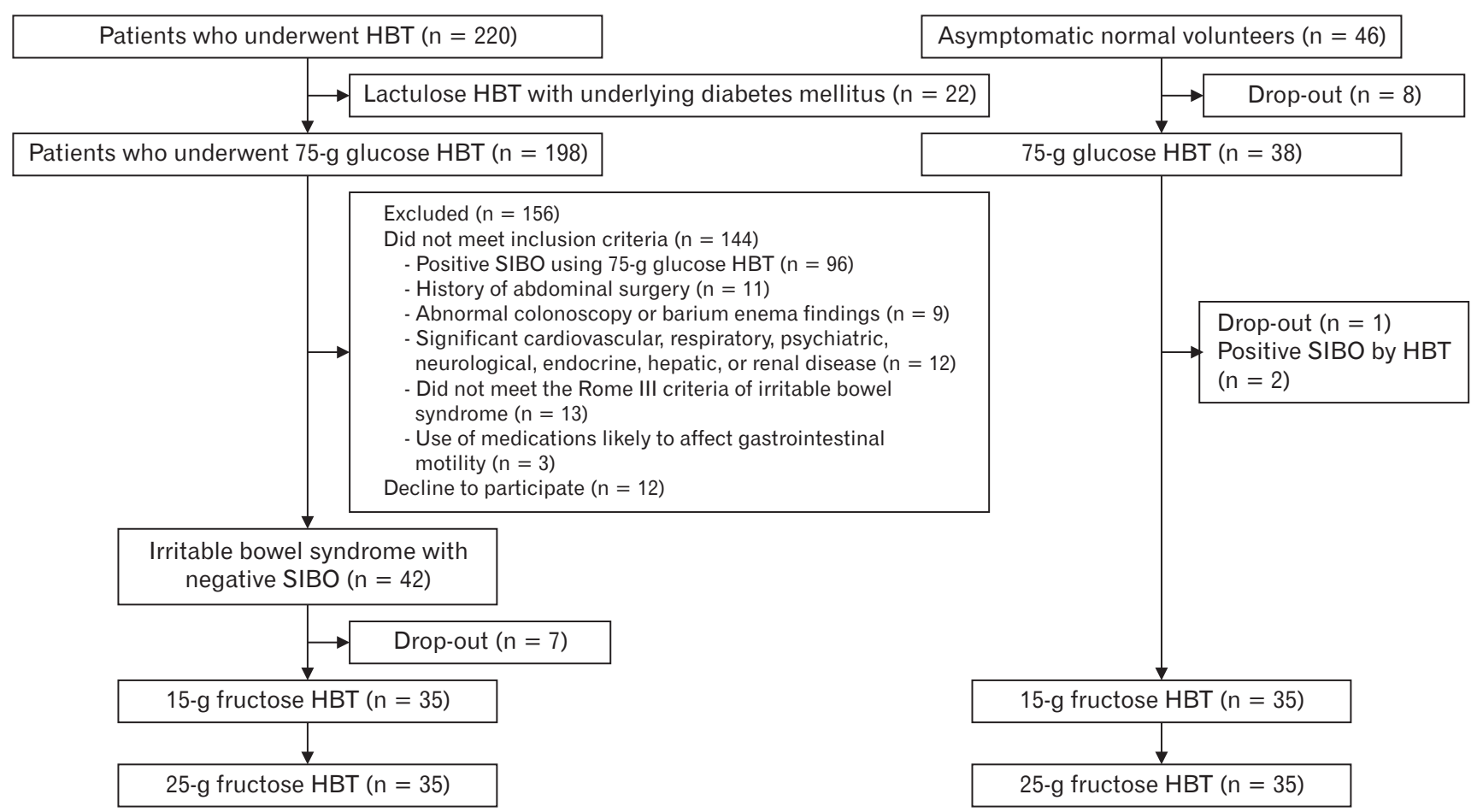

Figure 1. Allocation of study participants between the irritable bowel syndrome (IBS) and the asymptomatic control groups. HBT, hydrogen breath testing; SIBO, small intestinal bacterial overgrowth. 
ing, and flatulence (all $P<0.05$ ) (Table 1). Moreover, there were no significant differences among the different IBS subtypes in relation to the symptom reported, with 4 of the 10 (40.0\%) individuals with IBS-C, 2 of the 18 (11.1\%) individuals with IBS-D, and 1 of the $7(14.3 \%)$ individuals with IBS-M reporting symptoms $(P=$ $0.200)$.

\section{Hydrogen Breath Testing With 25-g Fructose}

The 25-g fructose HBT yielded positive results in 16 of the 35 (45.7\%) SIBO-negative patients with IBS and in 8 of the $35(22.9 \%)$ asymptomatic control individuals $(P=0.040)$ (Table 1, Fig. 2 and 3 ). The numbers of individuals reporting some of the symptoms associated with FM were significantly higher in the IBS group compared with the control group, and included abdominal pain, bloating,

Table 1. Characteristics of Patients With Irritable Bowel Syndrome and Asymptomatic Control Individuals and Their Hydrogen Breath Test Results

\begin{tabular}{|c|c|c|c|}
\hline Category & IBS individuals $(\mathrm{n}=35)$ & Asymptomatic controls $(\mathrm{n}=35)$ & $P$-value \\
\hline Female (n [\%]) & $19(54.3)$ & $17(48.6)$ & 0.810 \\
\hline Age $($ mean $\pm \mathrm{SD}, \mathrm{yr})$ & $42.7 \pm 13.8$ & $38.2 \pm 11.0$ & 0.160 \\
\hline Positive $15-\mathrm{g}$ fructose $\mathrm{HBT}$ result (n [\%]) & $7(20.0)$ & $2(5.7)$ & 0.070 \\
\hline \multicolumn{4}{|l|}{ 15-g fructose HBT (min) } \\
\hline Onset $\mathrm{H}_{2}$ & $12.0 \pm 27.4$ & $3.4 \pm 12.1$ & 0.150 \\
\hline Peak $\mathrm{H}_{2}$ & $13.7 \pm 32.0$ & $5.1 \pm 18.5$ & 0.170 \\
\hline Onset $\mathrm{CH}_{4}$ & $6.0 \pm 19.0$ & $0.9 \pm 5.1$ & 0.160 \\
\hline Peak $\mathrm{CH}_{4}$ & $11.1 \pm 33.4$ & $1.7 \pm 10.1$ & 0.160 \\
\hline Methane producer (n [\%]) & $2(5.7)$ & $2(5.7)$ & $>0.999$ \\
\hline \multicolumn{4}{|l|}{ Associated symptoms (n [\%]) } \\
\hline Abdominal pain & $8(22.9)$ & $2(5.7)$ & 0.040 \\
\hline Cramping & $2(5.7)$ & $2(5.7)$ & $>0.999$ \\
\hline Bloating & $14(40.0)$ & $5(14.3)$ & 0.020 \\
\hline Fullness & $11(31.4)$ & $4(11.4)$ & 0.040 \\
\hline Nausea & $7(20.0)$ & $6(17.1)$ & 0.760 \\
\hline Belching & $16(45.7)$ & $8(22.9)$ & 0.040 \\
\hline Ingestion & $3(8.6)$ & $0(0)$ & 0.070 \\
\hline Diarrhea & $5(14.3)$ & $1(2.9)$ & 0.090 \\
\hline Flatulence & $15(42.9)$ & $7(20.0)$ & 0.040 \\
\hline Positive $25-\mathrm{g}$ fructose $\mathrm{HBT}$ result (n [\%]) & $16(45.7)$ & $8(22.9)$ & 0.040 \\
\hline \multicolumn{4}{|l|}{ 25-g fructose HBT (min) } \\
\hline Onset $\mathrm{H}_{2}$ & $25.7 \pm 32.7$ & $14.6 \pm 30.3$ & 0.090 \\
\hline Peak $\mathrm{H}_{2}$ & $38.6 \pm 46.3$ & $31.7 \pm 57.7$ & 0.260 \\
\hline Onset $\mathrm{CH}_{4}$ & $2.6 \pm 11.2$ & $6.0 \pm 25.0$ & 0.950 \\
\hline Peak $\mathrm{CH}_{4}$ & $6.9 \pm 29.2$ & $7.7 \pm 32.0$ & 0.990 \\
\hline Methane producer (n [\%]) & $2(5.7)$ & $2(5.7)$ & $>0.999$ \\
\hline \multicolumn{4}{|l|}{ Associated symptoms (n [\%]) } \\
\hline Abdominal pain & $10(28.6)$ & $0(0)$ & $<0.010$ \\
\hline Cramping & $4(11.4)$ & $0(0)$ & 0.020 \\
\hline Bloating & $14(40.0)$ & $5(14.3)$ & 0.020 \\
\hline Fullness & $13(37.1)$ & $5(14.3)$ & 0.030 \\
\hline Nausea & $8(22.9)$ & $11(31.4)$ & 0.420 \\
\hline Belching & $16(45.7)$ & $13(37.1)$ & 0.470 \\
\hline Indigestion & $5(14.3)$ & $0(0)$ & 0.020 \\
\hline Diarrhea & $8(22.9)$ & $1(2.9)$ & 0.010 \\
\hline Flatulence & $18(51.4)$ & $7(20.0)$ & $<0.010$ \\
\hline
\end{tabular}

IBS, irritable bowel syndrome; HBT, hydrogen breath testing; $\mathrm{H}_{2}$, hydrogen; $\mathrm{CH}_{4}$, methane. 

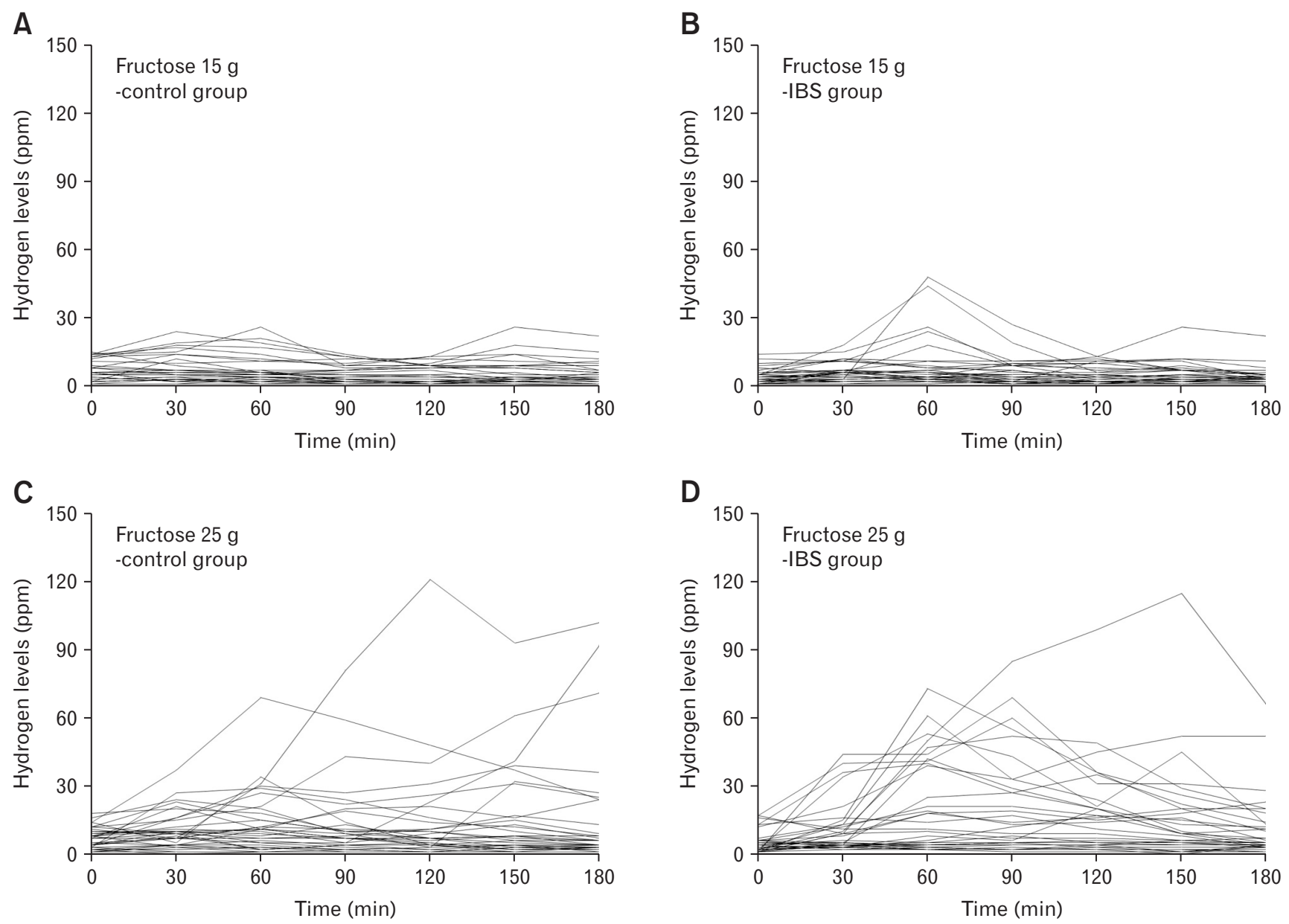

Figure 2. Hydrogen concentrations, expressed as parts per million ( $\mathrm{ppm}$ ), in the expired air of individuals following the ingestion of different fructose loads dissolved in $250 \mathrm{~mL}$ water. (A) $15 \mathrm{~g}$ of fructose ingested by the asymptomatic control group. (B) $15 \mathrm{~g}$ of fructose ingested by the group of patients with irritable bowel syndrome (IBS). (C) $25 \mathrm{~g}$ of fructose ingested by the asymptomatic control group. (D) $25 \mathrm{~g}$ of fructose ingested by the group of patients with IBS.

A

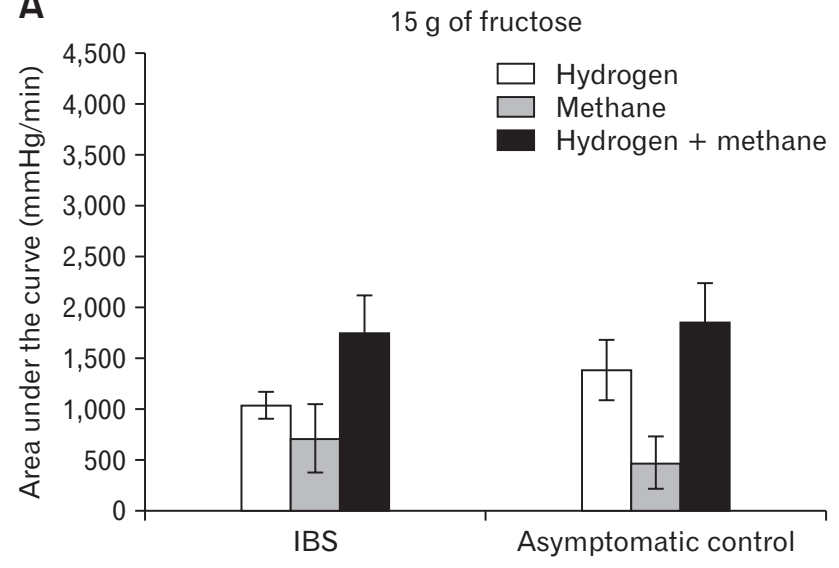

B

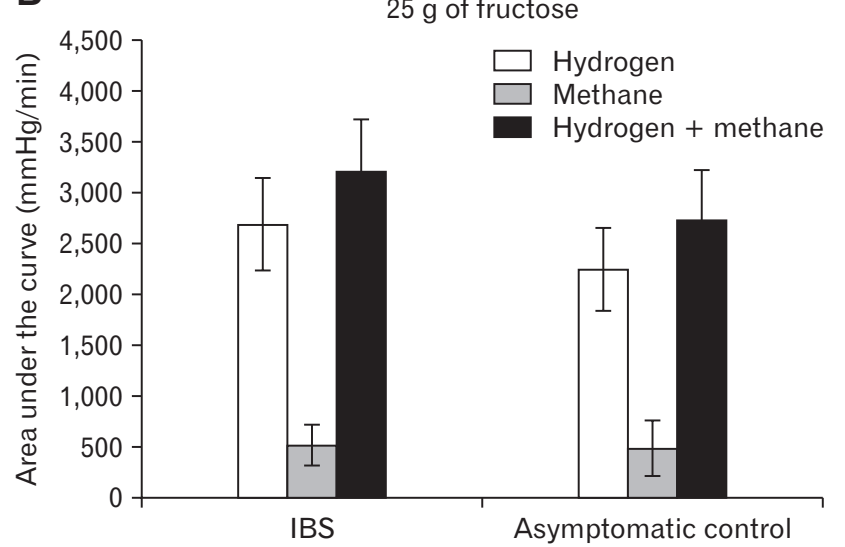

Figure 3. The areas under the curves of hydrogen and methane concentrations, expressed as $\mathrm{mmHg} / \mathrm{minute}$ and plotted as the means $( \pm$ standard errors), following the ingestion of (A) $15 \mathrm{~g}$ of fructose dissolved in $250 \mathrm{~mL}$ water and (B) $25 \mathrm{~g}$ of fructose dissolved in $250 \mathrm{~mL}$ water. IBS, irritable bowel syndrome. 
Table 2. Calories and Nutrient Intakes in Patients With Irritable Bowel Syndrome and Asymptomatic Control Individuals

\begin{tabular}{lccc}
\hline \multicolumn{1}{c}{ Mean intake } & IBS individuals $(\mathrm{n}=33)$ & Asymptomatic controls $(\mathrm{n}=28)$ & $P$-value \\
\hline Total calories $(\mathrm{kcal})$ & $2267.62 \pm 891.53$ & $1908.66 \pm 521.25$ & 0.150 \\
Total proteins $(\mathrm{g})$ & $80.72 \pm 33.75$ & $72.07 \pm 24.41$ & 0.380 \\
Vegetable protein $(\mathrm{g})$ & $49.70 \pm 23.90$ & $37.63 \pm 12.06$ & 0.050 \\
Animal protein $(\mathrm{g})$ & $31.02 \pm 18.24$ & $34.44 \pm 17.48$ & 0.340 \\
Total lipids $(\mathrm{g})$ & $45.27 \pm 20.22$ & $45.84 \pm 17.52$ & 0.550 \\
Vegetable lipids $(\mathrm{g})$ & $22.93 \pm 11.99$ & $19.11 \pm 7.18$ & 0.300 \\
Animal lipids $(\mathrm{g})$ & $22.33 \pm 11.74$ & $26.73 \pm 12.48$ & 0.120 \\
Total carbohydrates $(\mathrm{g})$ & $371.62 \pm 158.48$ & $290.47 \pm 89.14$ & 0.070 \\
Fructose $(\mathrm{g})$ & $75.60 \pm 51.83$ & $70.97 \pm 23.97$ & 0.390 \\
Fructose/total carbohydrate $(\%)$ & $22.29 \pm 13.29$ & $25.18 \pm 7.93$ & 0.070 \\
Total fiber $(\mathrm{g})$ & $21.24 \pm 11.35$ & $15.87 \pm 7.07$ & 0.040 \\
Ash $(\mathrm{g})$ & $22.52 \pm 14.56$ & $19.02 \pm 7.63$ & 0.640 \\
\hline
\end{tabular}

IBS, irritable bowel syndrome.

Values presented are mean $\pm \mathrm{SD}$.

fullness, indigestion, diarrhea, and flatulence (all $P<0.05$ ) (Table 1). Furthermore, there were no significant differences among the different IBS subtypes in relation to the symptoms reported, with 4 of the $10(40.0 \%)$ individuals with IBS-C, 9 of the $18(50.0 \%)$ individuals with IBS-D, and 3 of the 7 (42.9\%) individuals with IBS$\mathrm{M}$ reporting symptoms $(P=0.870)$.

\section{Dietary Habits}

Computer-aided nutritional analysis of the responses to the FFQ showed there were no significant between-group differences in relation to the mean total calorie intakes, and protein, lipid, carbohydrate, and fructose consumption (Table 2). However, the mean fiber intake was significantly higher in the IBS group than in the asymptomatic control individuals $(21.24 \pm 11.35 \mathrm{~g}$ vs $15.87 \pm 7.07$ g; $P=0.040)$ (Table 2).

\section{Adverse Effects}

Neither the 15-g nor the 25-g fructose HBTs was associated with any serious adverse effects.

\section{Discussion}

The indications for HBT are carbohydrate malabsorption, SIBO, the oro-cecal transit time, and other abdominal gas-related symptoms. ${ }^{11}$ The factors that influence carbohydrate malabsorption are the dosage of carbohydrate used during HBT, small bowel transit time, associated SIBO component, and enzymatic digestive capacity for the carbohydrate used. We tried to define the optimal dosage of fructose using different dosages of $15 \mathrm{~g}$ and $25 \mathrm{~g}$. More- over, to minimize the false-positive rate of FM caused by SIBO, subjects who showed positive results on $75-\mathrm{g}$ glucose HBT were excluded from the study design. Finally, lactulose can accelerate the small bowel transit based on combined oro-cecal scintigraphy and lactulose HBT. ${ }^{18}$ Therefore, glucose HBT was used in this study.

HBT, using glucose or lactulose, is the most widely available indirect test for SIBO. ${ }^{11,19}$ Recently, lactulose was found to measure colonic bacterial fermentation rather than distal SIBO, ${ }^{18}$ rendering glucose-based HBTs more suitable for assessing SIBO. ${ }^{11}$ Consequently, we utilized glucose rather than lactulose in the SIBO test.

Despite glucose having a better discriminative ability than lactulose in the diagnosis of SIBO, the limitation of using glucose is that it only determines the presence of SIBO within the proximal small bowel and it does not detect SIBO in the distal small bowel. ${ }^{11}$ Moreover, the sensitivity and specificity of glucose in the diagnosis of SIBO are $20-93 \%$ and 30-86\%, respectively. ${ }^{11,20,21}$ However, we tried to gather people without SIBO using a clinically available test with a higher specificity; glucose based HBT was chosen rather than lactulose based HBT. There have been trials using several concentrations of glucose based HBTs in the detection of SIBO. ${ }^{22-24}$ Among these, $50 \mathrm{~g}$ or $75 \mathrm{~g}$ of glucose were frequently used. Compared to the $50 \mathrm{~g}$ of glucose, $75 \mathrm{~g}$ of glucose is thought to explain distal small bowel SIBO compared to the $50 \mathrm{~g}$ of glucose. ${ }^{11}$ Therefore, we chose the $75 \mathrm{~g}$ of glucose in this study. We believe that the false-positive FM rate associated with SIBO was largely excluded from this study. Furthermore, this is the first study that compares the prevalence of FM in patients with IBS with that in asymptomatic control individuals, while excluding SIBO.

The optimal dosage of fructose for FM diagnosis is unclear, ${ }^{11}$ 
although a recent study using different doses showed that $25 \mathrm{~g}$ of fructose mixed in $250 \mathrm{~mL}$ of water was effective in FM diagnosis. ${ }^{8}$ However, that study was performed only in Western subjects. Recently, in a published paper based on Indian people, FM was reported in up to $14 \%$ of IBS patients using $25 \mathrm{~g}$ of fructose dissolved in $250 \mathrm{~mL}$ of water. ${ }^{25}$ However, there have been no data in Korean people until now. We postulated that the effective fructose dosage for FM diagnosis may differ between Asian and Western subjects; hence, we tested $15 \mathrm{~g}$ and $25 \mathrm{~g}$ of fructose mixed in $250 \mathrm{~mL}$ of water ( $6 \%$ and $10 \%$, respectively). The 15 -g fructose HBT showed that subjects with FM tended to be in the IBS group rather than in the asymptomatic control group, but this difference was not statistically significant. In contrast, the 25 -g fructose HBT showed a statistically significant difference in FM between the 2 study groups. Because of financial limitations, we could not perform HBTs using other fructose doses, for example, 20-g or 30-g fructose. Because the concentration as well as the dose may affect fructose absorption, it is worth testing $15 \mathrm{~g}$ and $25 \mathrm{~g}$ of fructose with higher or lower concentrations. However, we could not evaluate other concentrations because of financial and time limitations.

Despite our inability to determine the optimal dose, our findings suggest that $25 \mathrm{~g}$ of fructose is an appropriate dose to clearly differentiate between patients with IBS and asymptomatic control individuals in relation to $\mathrm{FM}$.

The $25 \mathrm{~g}$ fructose HBT yielded positive results for FM in 16 of the 35 (45.7\%) SIBO-negative patients with IBS and in 8 of the 35 (22.9\%) asymptomatic control individuals $(P=0.040)$. Symptoms experienced during the HBTs were significantly more frequent in the IBS group than in the control group, and these symptoms included abdominal pain, bloating, fullness, indigestion, diarrhea, and flatulence ( $P<0.05$ for each symptom), all of which are typical symptoms of IBS. These findings provide further evidence of the association between FM and IBS. Our study used different doses of fructose in the detection of FM. However, even healthy volunteers showed a high number of positivity with $25 \mathrm{~g}$ of fructose $(22.9 \%$ [2/35]), not with $15 \mathrm{~g}$ of fructose $(5.7 \%[8 / 35])$. This finding supports that healthy subjects in Korea might have the capacity to absorb up to a dose of 25-g fructose, although the absorption ability is variable in each subject. Using different concentrations in this study may be one of the reasons of this difference, and this could be a limitation and needs caution in the interpretation of results. Nevertheless, we believe that the high positivity of $25-\mathrm{g}$ fructose HBT in patients with IBS may suggest the correlation between FM and IBS.

We do not know the exact reason for this difference compared to previous Western papers. ${ }^{8}$ However, we postulate that there may be differences in absorptive ability by race or dietary patterns in Eastern and Western cultures. Moreover, this study was based on a relatively small number of participants. Therefore, our findings may need further validation with a larger number of IBS patients and asymptomatic control individuals. HBTs using pure fructose may over-diagnose FM because most people ingest mixtures of glucose and fructose, also known as HFCS, and glucose assists the absorption of fructose by the small intestine. ${ }^{26}$ However, we measured FM in both the IBS and the control groups, thereby offsetting the effects of over-estimation in the IBS group.

There were conflicting results with regard to if patients with IBS have a more likelihood of FM (40-80\%) as compared to asymptomatic control individuals (11-70\%). ${ }^{8}$ One study showed that the prevalence of $25-\mathrm{g}$ fructose malabsorption is higher in patients with functional bowel disorders (36-75\%) than healthy controls (0$50 \%) .{ }^{26}$ Several possible mechanisms for the higher proportion of FM in patients with IBS include an altered enteric microbiome, increased intestinal permeability, rapid small bowel transit, and immune responsiveness. ${ }^{27,28}$ These pathogenic mechanisms could affect fructose transporters in patients with IBS. The other possible mechanism was that intestinal glucose transporters are injured and this would then result in FM.

Analysis of the responses to the $\mathrm{FFQ},{ }^{13}$ showed that fructose intake did not differ significantly between the 2 groups. Although the FFQ has been used in many studies, ${ }^{14,29,30}$ the questionnaire's categories only assess intakes of typical essential nutrients, including protein, lipid, carbohydrate, and vitamins. Given that the currently used FFQ does not include fructose intakes, the dietitian (Y-O.P.) assessed the fructose content of the 103 food items covered by the FFQ. While we know from previously published papers that many foods use HFCS, and high fructose levels are present in, for example, soda, apple juices, grape juices, and beers, fructose levels remain undetermined in popular Korean ethnic foods, including traditional noodles with hot spices, cakes, or cookies flavored with corn syrup, and fried chicken flavored with traditional Korean spices. Many Korean foods contain spices, and HFCS may replace particular food additives. Furthermore, a validated questionnaire in the Korean language has yet to be designed to assess the fructose content of diets. Moreover, some study participants responded incorrectly to questions by mistake. Consequently, not all of the answers could be included in the final assessment and analysis. Recently, a published review article showed Korean dishes including kimchi and doenjang may have high fermentable oligo-, di-, and mono-saccharides; and polyols (FODMAP) ingredients. ${ }^{31}$ We think that our study may be 
meaningful as a stepping stone to elicit the correlation between IBS symptoms and food, especially FODMAP.

Interestingly, fiber intake was significantly higher in the group of participants with IBS than in the control group. Fiber has been used to treat various gastrointestinal disorders, including constipation, ulcerative colitis, and even obesity, ${ }^{32}$ but it is also known to exacerbate problems, including abdominal distension, flatulence, and diarrhea. ${ }^{32}$ Although the role of fiber in IBS is unclear, these previously cited studies have been performed in Western rather than in Asian countries. On the basis of this result, fiber intake appears to be judicious, especially in patients who have IBS.

FM and SIBO have a direct cause and effect relationship. ${ }^{1}$ Fructose may promote the survival of bacteria in the distal small intestine by providing a substrate for the synthesis of fructans as adherence factors. ${ }^{1}$ While there is no direct evidence that this occurs in the distal small intestine, IBS symptoms improve when all potential metabolic substrates for bacteria are removed by feeding patients elemental diets. ${ }^{33}$ Furthermore, antibiotic therapy abolished FM in a high proportion of patients who had presumed SIBO, which was concomitant with reductions in symptoms. ${ }^{34}$ Interestingly, 2 of the asymptomatic control individuals showed positive results on the SIBO test. Additional studies using larger numbers of participants are required.

In conclusion, use of the $25-\mathrm{g}$ fructose HBT showed that the incidence of FM was significantly higher in SIBO-negative patients with IBS than in asymptomatic control individuals, suggesting that FM may be closely associated with IBS. Education regarding the dietary control of foods that contain fructose may be helpful in the management of patients with IBS.

Acknowledgements: This study was partially presented in Federation of Neurogastroenterology and Motility (FNM) 2014 (Neurogastroenterol Motil 2014;26(suppl 1):P-102) and Digestive Disease Week (DDW) 2014 (Gastroenterology 2014;146:S-221).

Financial support: This research was supported by a grant of the Korea Health Technology R\&D Project through the Korea Health Industry Development Institute(KHIDI), funded by the Ministry of Health \& Welfare, Republic of Korea (No. HI15C3078) and the Asian Institute for Life Sciences (No. 20110524).

\section{Conflicts of interest: None.}

Author contributions: Kee Wook Jung and Myeongsook Seo were responsible for the study from its design to writing the article; Young Hwan Cho founded the study design and helped with patient enrollment; In Ja Yoon and So Young Seo performed all of the tests; Young-Ok Park and So-Yoon Yoon collected the Food Frequency Questionnaires and analyzed the data; Jungbok Lee performed sample size calculation and statistical analysis; Seung-Jae Myung, Dong-Hoon Yang, Hyo Jeong Lee, Sang Hyoung Park, Kyung Jo Kim, Byong Duk Ye, Jeong-Sik Byeon, Hwoon-Yong Jung, Suk-Kyun Yang, and Jin-Ho Kim supervised the study and provided important intellectual contributions; the guarantors of the article are Kee Wook Jung and Seung-Jae Myung; and all authors approved the final version of the manuscript.

\section{References}

1. Gibson PR, Newnham E, Barrett JS, Shepherd SJ, Muir JG. Review article: fructose malabsorption and the bigger picture. Aliment Pharmacol Ther 2007;25:349-363.

2. Park YK, Yetley EA. Intakes and food sources of fructose in the United States. Am J Clin Nutr 1993;58(5 suppl):737S-747S.

3. Yom HW. Clinical nutrition therapy of metabolic syndrome in adolescents. Korean J Pediatr Gastroenterol Nutr 2011;14:350-358.

4. Densupsoontorn N, Jirapinyo P, Thamonsiri N, Wongam R. Fructose malabsorption in Thai adult. Asia Pac J Clin Nutr 2007;16:209-212.

5. Riby JE, Fujisawa T, Kretchmer N. Fructose absorption. Am J Clin Nutr 1993;58(5 suppl):748S-753S.

6. Skoog SM, Bharucha AE. Dietary fructose and gastrointestinal symptoms: a review. Am J Gastroenterol 2004;99:2046-2050.

7. Rumessen JJ, Gudmand-Høyer E. Absorption capacity of fructose in healthy adults. Comparison with sucrose and its constituent monosaccharides. Gut 1986;27:1161-1168.

8. Rao SS, Attaluri A, Anderson L, Stumbo P. Ability of the normal human small intestine to absorb fructose: evaluation by breath testing. Clin Gastroenterol Hepatol 2007;5:959-963.

9. Choi YK, Johlin FC Jr, Summers RW, Rao SS. Fructose intolerance: an under-recognized problem. Am J Gastroenterol 2003;98:1348-1353.

10. Pimentel M. An evidence-based treatment algorithm for IBS based on a bacterial/SIBO hypothesis: part 2. Am J Gastroenterol 2010;105:12271230.

11. Gasbarrini A, Corazza GR, Gasbarrini G, et al. Methodology and indications of H2-breath testing in gastrointestinal diseases: the Rome Consensus Conference. Aliment Pharmacol Ther 2009;29(suppl 1):1-49.

12. Longstreth GF, Thompson WG, Chey WD, Houghton LA, Mearian F, Spiller RC. Functional bowel disorders. Gastroenterology 2006;130:1480-1491.

13. Ahn Y, Kwon E, Shim JE, et al. Validation and reproducibility of food frequency questionnaire for Korean genome epidemiologic study. Eur J Clin Nutr 2007;61:1435-1441.

14. Cho YA, Shin A, Kim J. Dietary patterns are associated with body mass 
index in a Korean population. J Am Diet Assoc 2011;111:1182-1186.

15. Hejazi RA, McCallum RW. Breath tests for small intestinal bacterial overgrowth. In: Parkman HP, McCallum RW, Rao SCC, eds. GI motility testing Thorofare: Slack 2011:145-150.

16. Attaluri A, Rao SSC. Breath testing for carbohydrate intolerance. In: Parkman HP, McCallum RW, Rao SSC, eds. GI motility testing. Thorofare: Slack 2011:137-143.

17. Johlin FC Jr, Panther M, Kraft N. Dietary fructose intolerance: diet modification can impact self-rated health and symptom control. Nutr Clin Care 2004;7:92-97.

18. Yu D, Cheeseman F, Vanner S. Combined oro-caecal scintigraphy and lactulose hydrogen breath testing demonstrate that breath testing detects oro-caecal transit, not small intestinal bacterial overgrowth in patients with IBS. Gut 2011;60:334-340.

19. Simren M, Stotzer PO. Use and abuse of hydrogen breath tests. Gut 2006;55:297-303.

20. Saad RJ, Chey WD. Breath testing for small intestinal bacterial overgrowth: maximizing test accuracy. Clin Gastroenterol Hepatol 2014;12:1964-1972.

21. Ghoshal UC, Ghoshal U. Small intestinal bacterial overgrowth and other intestinal disorders. Gastroenterol Clin North Am 2017;46:103-120.

22. King CE, Toskes PP. Comparison of the 1-gram $\left[{ }^{14} \mathrm{C}\right]$ xylose, 10 -gram lactulose- $\mathrm{H}_{2}$, and 80 -gram glucose- $\mathrm{H} 2$ breath tests in patients with small intestine bacterial overgrowth. Gastroenterology 1986;91:1447-1451.

23. Bauer TM, Schwacha H, Steinbrückner B, et al. Diagnosis of small intestinal bacterial overgrowth in patients with cirrhosis of the liver: poor performance of the glucose breath hydrogen test. J Hepatol 2000;33:382386.

24. Ghoshal UC, Ghoshal U, Das K, Misra A. Utility of hydrogen breath tests in diagnosis of small intestinal bacterial overgrowth in malabsorption syndrome and its relationship with oro-cecal transit time. Indian J Gastro- enterol 2006;25:6-10

25. Sharma A, Srivastava D, Verma A, Misra A, Ghoshal UC. Fructose malabsorption is not uncommon among patients with irritable bowel syndrome in India: a case-control study. Indian J Gastroenterol 2014;33:466470.

26. Skoog SM, Bharucha AE, Zinsmeister AR. Comparison of breath testing with fructose and high fructose corn syrups in health and IBS. Neurogastroenterol Motil 2008;20:505-511.

27. Kennedy PJ, Clarke G, Quigley EM, et al. Gut memories: towards a cognitive neurobiology of irritable bowel syndrome. Neurosci Biobehav Rev 2012;36:310-340.

28. Ohman L, Simrén M. Pathogenesis of IBS: role of inflammation, immunity and neuroimmune interactions. Nat Rev Gastroenterol Hepatol 2010;7:163-173.

29. Cho ER, Shin A, Lim SY, Kim J. Dietary patterns and their associations with health behaviours in Korea. Public Health Nutr 2011;14:356-364.

30. Cho YA, Kim J, Cho ER, Shin A. Dietary patterns and the prevalence of metabolic syndrome in Korean women. Nutr Metab Cardiovasc Dis 2011;21:893-900.

31. Iacovou M, Tan V, Muir JG, Gibson PR. The low FODMAP diet and its application in east and southeast asia. J Neurogastroenterol Motil 2015;21:459-470.

32. Eswaran S, Muir J, Chey WD. Fiber and functional gastrointestinal disorders. Am J Gastroenterol 2013;108:718-727.

33. Pimentel M, Constantino T, Kong Y, Bajwa M, Rezaei A, Park S. A 14day elemental diet is highly effective in normalizing the lactulose breath test. Dig Dis Sci 2004;49:73-77.

34. Nucera G, Gabrielli M, Lupascu A, et al. Abnormal breath tests to lactose, fructose and sorbitol in irritable bowel syndrome may be explained by small intestinal bacterial overgrowth. Aliment Pharmacol Ther 2005;21:1391-1395. 